\title{
ANÁLISIS DE CORRESPONDENCIA DE LA POBREZA MULTIDIMENSIONAL EN LOS DISTRITOS DE LA PROVINCIA DEL SANTA, 2017
}

\author{
CORRESPONDENCE ANALYSIS OF MULTIDIMENSIONAL \\ POVERTY IN THE DISTRICTS OF THE PROVINCE OF \\ SANTA, 2017 \\ iD Luis Alberto Calderón Yarlequé \\ 1Universidad Nacional del Santa, Ancash, Perú.
}

Correspondencia:

Mag. Luis Alberto Calderón Yarlequé

lcalderon@uns.edu.pe

\section{RESUMEN}

El presente trabajo de investigación, tuvo como objetivo evaluar la pobreza multidimensional en la Provincia del Santa con la información de la Encuesta Nacional de Hogares 2017, para lo cual se seleccionó una nuestra de 477 registros. La pobreza multidimensional se evaluó mediante el método de las necesidades básicas insatisfechas: no pobres (cuenta con todas las necesidades básicas), regularmente pobre (al menos no cuenta con una necesidad básica), pobres (al menos no cuenta con dos o tres necesidades básicas), pobreza extrema (vive en escenarios difíciles), utilizándose como punto de corte para el índice de pobreza multidimensional > 5.446. Se aplicó el Análisis de Correspondencia Múltiple (ACM) y la correlación de Spearman. El 1,7 \% eran no pobres; $85,5 \%$, regularmente pobre; $12,4 \%$ pobres y 0,4 \% en pobreza extrema. No hubo correlación entre las dimensiones educación y salud de las familias, ni entre las dimensiones educación y bienestar de infraestructura de las familias de la provincia del Santa. Hubo una correlación moderada entre dimensiones educación y bienestar de servicios 0.567 . El índice de pobreza multidimensional fue 5.446, clasificándose los hogares como pobres.

Palabras clave: pobreza multidimensional, análisis de correspondencia múltiple, necesidades básicas insatisfechas.

\section{ABSTRACT}

The objective of this research work was to evaluate multidimensional poverty in the Province of Santa with the information from the 2017 National Household Survey, for which one of our 477 records was selected. Multidimensional poverty was assessed using the unsatisfied basic needs method: not poor (has all basic needs), regularly poor (at least does not have one basic need), poor (at least does not have two or three basic needs), extreme poverty (lives in difficult scenarios), being used as a cutoff point for the multidimensional poverty index> 5.446. Multiple Correspondence Analysis (MCA) and Spearman's correlation were applied. $1.7 \%$ were non-poor; 85.5 
$\%$, regularly poor; $12.4 \%$ poor and $0.4 \%$ in extreme poverty. There was no correlation between the education and health dimensions of the families, nor between the education and infrastructure well-being dimensions of the families in the province of Santa. There was a moderate correlation between dimensions education and wellbeing of services 0.567 . The multidimensional poverty index was 5,446 , classifying households as poor.

Key words: multidimensional poverty, multiple correspondence analysis, unsatisfied basic needs.

\section{INTRODUCCIÓN}

A lo largo del tiempo uno de los métodos para la identificación de la pobreza ha sido el de Necesidades Básicas Insatisfechas (NBI), desde el año 1981 por Paul Streeten e implementada por la Comisión Económica para América Latina y el Caribe (CEPAL). Seguidamente, la pobreza monetaria apoya como complemento a las Necesidades Básicas Insatisfechas para generar una nueva tipología, para tener en cuenta el posible estado transitorio o crónico de la pobreza. Desde 1990, los resultados de Desarrollo Humano incluyen el Índice de Desarrollo Humano (IDH) (Kaztman, 1989).

Expandiéndose en América Latina, en la segunda mitad de 1980, tanto la elaboración de mapas de pobreza, a partir del método de $\mathrm{NBI}$, aplicado la información de los censos de población, como la medición de la pobreza de ingresos, Beccaria y Minujín en Argentina y Kaztman en Uruguay indagaron si con ambos métodos se obtenían incidencias homogéneas de pobreza y se identificaban los mismos hogares como pobres; para ello elaboraron cuadros de contingencia y encontraron respuestas negativas, transcurrido el tiempo (Boltvinik, 2013).

El Programa de las Naciones Unidas para el Desarrollo (PNUD) y la Iniciativa de Pobreza y Desarrollo Humano de la Universidad de Oxford publicaron recientemente el Informe del Índice Global de Pobreza Multidimensional 2018 (MPI 2018). Para su elaboración, se utiliza el perfil de las privaciones que experimenta cada individuo en los 10 indicadores que lo conforman distribuidos en tres dimensiones fundamentales: salud, educación y estándares de vida, buscando identificar las múltiples maneras en las que un individuo experimenta la pobreza. El índice identifica a una persona como pobre multidimensional si se ve privada de al menos un tercio de los indicadores evaluados (Castañeda, 2018).

Según Morales (2004), "para todo análisis de datos sociológicos es un oficio en el que se debe tener una relevancia estricta las herramientas estadísticas y con el uso de los programas estadísticos que posibilitan hacer un análisis con cierta facilidad y rapidez los problemas en grandes bases de datos, por lo tanto, el analista se concentra cada vez más en el estudio de la interpretación sobre las técnicas estadísticas".

"Cada vez son más necesarias las aplicaciones de las técnicas estadísticas para más de dos variables categóricas (nominal, ordinal), y llamado análisis de correspondencias múltiples (ACM), no es un tema muy aplicado ni bien definido, presentándose en las diferentes literaturas de enfoques para su procesamiento en sus respetivos tratamientos". (Greenacre,1984).

Según Saavedra (2012), "El Análisis de Correspondencia Múltiple (ACM) se basa en la aplicación del análisis de correspondencia a la denominada matriz de Burt, la cual no es sino una matriz cuadrada simétrica formada por las tablas de contingencia del cruce de todos los pares de variables, en donde en la diagonal se hallan los cruces de las variables con ellas mismas. Esta codificación hace que los porcentajes de inercia de las dimensiones de los mapas en el ACM sean artificialmente bajos, lo que conlleva a una subestimación de la calidad de un análisis multidimensional. Al Análisis de Correspondencia Múltiple se le conoce como HOMALS (busca homogeneidad en las variables u objetos que agrupa y heterogeneidad entre los distintos grupos). (Castañeda, 2018). El objetivo general de esta investigación fue evaluar la 
pobreza multidimensional según el análisis de correspondencia múltiple (ACM) en la provincia Del Santa, 2017.

\section{MATERIALES Y MÉTODOS}

Se trata de un estudio descriptivo, con diseño no experimental, transversal y retrospectivo en la pobreza multidimensional.

La población estuvo constituida por 726 hogares de la provincia del Santa en condiciones bajas y muy bajas de pobreza en los años 2017.

\section{Tabla 1}

\section{Población de la provincia del Santa por categoría}

\begin{tabular}{lcc}
\hline \multicolumn{1}{c}{ Categoría } & Cantidad & Proporción \\
\hline INVASIONES & 351 & 0.50 \\
ASENTAMIENTOS HUMANOS & 257 & 0.40 \\
PUEBLOS JÓVENES & 118 & 0.10 \\
TOTAL & 726 & \\
\hline
\end{tabular}

Fuente: INEI-2017 -2018

La muestra estuvo constituida por hogares 344 hogares del Distrito del Santa, en el período Enero - diciembre 2017 - 2018. El nivel de confianza de los resultados muéstrales de un 95\%. (INEI, 2017,2018).
Se determinó el tamaño de muestra usando el muestreo aleatorio Estratificado con afijación proporcional y el cálculo fue de la siguiente forma:

$$
\begin{gathered}
n_{0}=\frac{P * Q * Z^{2}}{E^{2}}=\frac{0.189 * 0.811 * 1.96^{2}}{0.03^{2}}=654 \\
n=\frac{n_{o}}{1+\frac{n_{o}}{N}}=\frac{654}{1+\frac{654}{726}}=477 \text { Hogares }
\end{gathered}
$$

Donde:

$\mathrm{P}=$ indice de pobreza estimada en la proviancia del santa en el 2017 - 2018 en base "perú: población y condición de pobreza, según departamento, provincia y distrito

$\mathrm{Q}=$ indice de no pobreza

$\mathrm{Z}=$ nivel de Confianza al 95\%

$\mathrm{E}=$ Margen de error 3\%

no= Tamaño de muestra estratificada

$\mathrm{N}=$ poblacion de hogares 
Tabla 2

Muestra por estrato de pobreza multidimensional en la provincia del Santa 2017 - 2018

\begin{tabular}{lccc}
\hline \multicolumn{1}{c}{ Categoría } & Cantidad & Proporción & Muestra Estratificada \\
\hline Invasiones & 351 & 0.48 & 166 \\
Asentamientos humanos & 257 & 0.35 & 122 \\
Pueblos jóvenes & 118 & 0.16 & 56 \\
TOTAL & 726 & & 477 \\
\hline
\end{tabular}

\section{RESULTADOS}

\section{Tabla 3}

Nivel de la pobreza multidimensional según el análisis de correspondencia múltiple (ACM) en la provincia del santa, 2017

\begin{tabular}{cccccccccc}
\hline & \multicolumn{9}{c}{ Nivel de Pobreza } \\
Distritos & Aceptable & \multicolumn{2}{c}{ Regular } & \multicolumn{2}{c}{ Pobres } & Pobre Extremo & Población \\
& $\mathrm{n}$ & $\%$ & $\mathrm{n}$ & $\%$ & $\mathrm{n}$ & $\%$ & $\mathrm{n}$ & $\%$ & \\
\hline Encuestada \\
Chimbote & 4 & 2,0 & 174 & 88,8 & 18 & 9,2 & 0 &, 0 & 196 \\
Nuevo Chimbote & 1 & 0,6 & 140 & 83,3 & 25 & 14,9 & 2 & 1,2 & 168 \\
Coishco & 1 & 3,8 & 23 & 88,5 & 2 & 7,7 & 0 &, 0 & 26 \\
Santa & 2 & 6,3 & 30 & 93,8 & 0 &, 0 & 0 &, 0 & 32 \\
Cáceres del Perú & 0 &, 0 & 11 & 68,8 & 5 & 31,3 & 0 &, 0 & 16 \\
Macate & 0 &, 0 & 6 & 75,0 & 2 & 25,0 & 0 &, 0 & 8 \\
Moro & 0 &, 0 & 0 &, 0 & 0 &, 0 & 0 &, 0 & 0 \\
Nepeña & 0 &, 0 & 20 & 80,0 & 5 & 20,0 & 0 &, 0 & 25 \\
Samanco & 0 &, 0 & 4 & 66,7 & 2 & 33,3 & 0 &, 0 & 6 \\
Media Pobreza & & 1.7 & & 85.5 & & 12.4 & & 0.4 &
\end{tabular}

Fuente: INEI/ENAHO 2017

El nivel de pobreza multidimensional en Áncash se comporta en 4 niveles aplicando el método de las necesidades básica, es aceptable en los Distritos de Chimbote, Coishco y Santa; Es considerado regularmente pobre todos los distritos a excepción de Moro; Se consideraron un nivel pobre todos menos Santa y Moro; y, por último, Pobre extremo es el distrito de Nuevo Chimbote. 


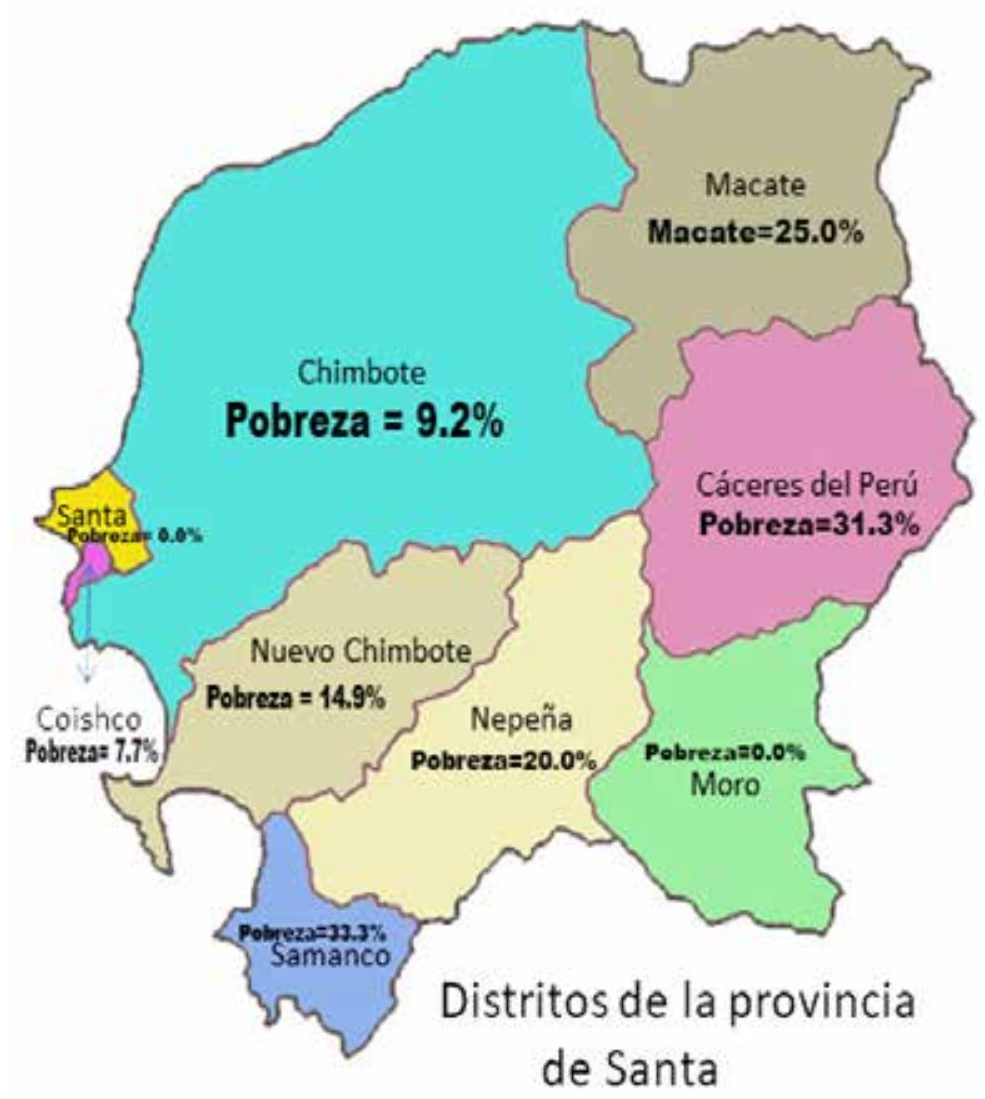

Figura 1. Nivel de la pobreza multidimensional según el análisis de correspondencia múltiple (ACM) en la provincia del Santa, 2017.

\section{Tabla 4}

Características de la vivienda y del hogar según bienestar de infraestructura, ENAHO 2017

\begin{tabular}{|c|c|c|c|c|}
\hline Dimensión & $\begin{array}{c}\text { Alfa de } \\
\text { Cronbach }\end{array}$ & $\begin{array}{c}\text { Varia } \\
\text { Total } \\
\text { (autovalor) }\end{array}$ & contabili & $\%$ de varianza \\
\hline 1 &, 723 & 2,370 &, 474 & 47,401 \\
\hline 2 &, 315 & 1,338 & ,268 & 26,751 \\
\hline 3 & 201 & 1,192 & ,238 & 23,843 \\
\hline Total & & 4,900 & ,980 & \\
\hline Media &, $485^{\mathrm{a}}$ & 1,633 &, 327 & 32,665 \\
\hline
\end{tabular}

a. La media de alfa de Cronbach se basa en la media de autovalor.

La Tabla 4, representa las características de la vivienda y del hogar según bienestar de infraestructura. Para la información del modelo es explicada por cada dimensión; permite analizar la importancia de cada una de ellas. Se puede observar que la primera dimensión es más importante para el modelo. Se espera que las dimensiones que se obtienen a raíz de análisis de correspondencia a mayor dependencia de las variables mayor inercia, esto quiere decir que las categorías presentan mayor dispersión de varianza en la primera dimensión y en las 
dimensiones 2 y 3 , son parecidas en cuanto a variabilidad.

\section{Tabla 5}

\section{Características de materiales en relación de tipo de vivienda}

\begin{tabular}{|c|c|c|c|c|c|}
\hline & $\begin{array}{l}\text { Tipo de } \\
\text { vivienda }\end{array}$ & $\begin{array}{c}\text { Material en las } \\
\text { Paredes }\end{array}$ & $\begin{array}{c}\text { Material en los } \\
\text { Pisos }\end{array}$ & $\begin{array}{c}\text { Material en los } \\
\text { Techos }\end{array}$ & $\begin{array}{l}\text { Habitaciones } \\
\text { en la Vivienda }\end{array}$ \\
\hline Tipo de vivienda & 1,000 &, 150 & , 166 &, 120 & ,094 \\
\hline Material en las paredes & ,150 & 1,000 & 635 & 577 & 241 \\
\hline Material en los pisos & , 166 & ,635 & 1,000 &, 548 & ,303 \\
\hline Material en los techos &, 120 &, 577 &, 548 & 1,000 & ,207 \\
\hline Habitaciones en la vivienda & ,094 & ,241 & ,303 & ,207 & 1,000 \\
\hline Dimensión & 1 & 2 & 3 & 4 & 5 \\
\hline Autovalor & 2,370 & 950 & 864 & ,457 & ,359 \\
\hline
\end{tabular}

En la Tabla 5, podemos observar una correlación de las variables transformadas entre el material predominante de los pisos y el material predominante en las paredes es el más alto, seguido de: el material predominante en los techos y el material predominante en las paredes es el segundo más alto y el material predominante en los techos es con el material predominante en los pisos es el tercero, respectivamente.

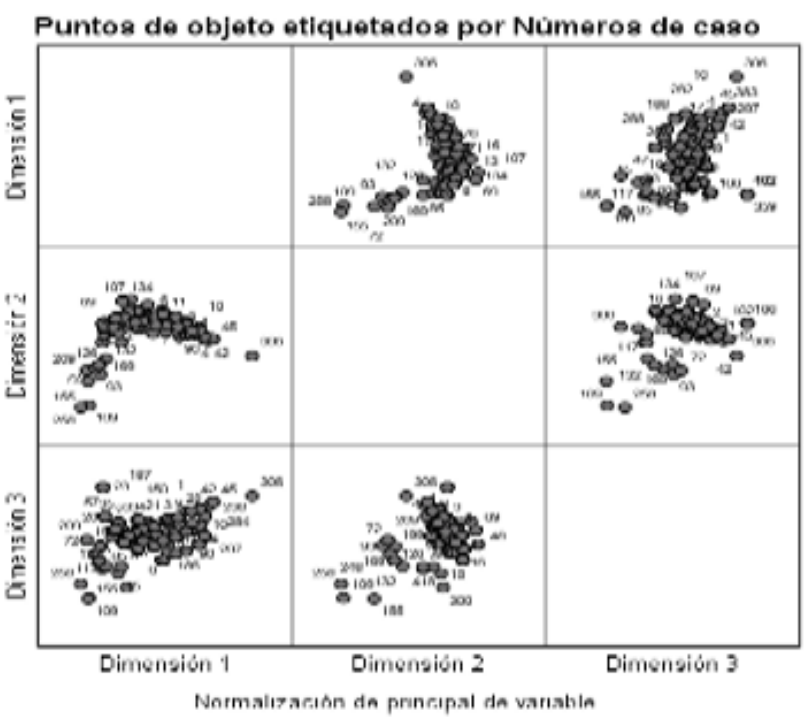

Figura 2. Gráfico de cuantificaciones para la característica de la vivienda y del hogar, ENAHO 2017. 
Tabla 6

Tipo de Vivienda y las características por dimensiones

\begin{tabular}{lcccc}
\hline & \multicolumn{3}{c}{ Dimensión } & \\
& 1 & 2 & 3 & Media \\
\hline Tipo de vivienda &, 088 &, 450 &, 219 &, 252 \\
El material en las paredes &, 710 &, 019 &, 152 &, 294 \\
El material en los pisos &, 719 &, 659 &, 421 &, 600 \\
El material en los techos &, 627 &, 170 &, 068 &, 288 \\
Habitaciones en la vivienda &, 226 &, 039 &, 332 &, 199 \\
Total activo & 2,370 & 1,338 & 1,192 & 1,633 \\
\% de varianza & 47,401 & 26,751 & 23,843 & 32,665 \\
\hline
\end{tabular}

En la Tabla 6, se observa que las medidas el de las paredes, pisos y techo, mientras que, en discriminantes en la primera dimensión fueron, la segunda dimensión, el material de piso.

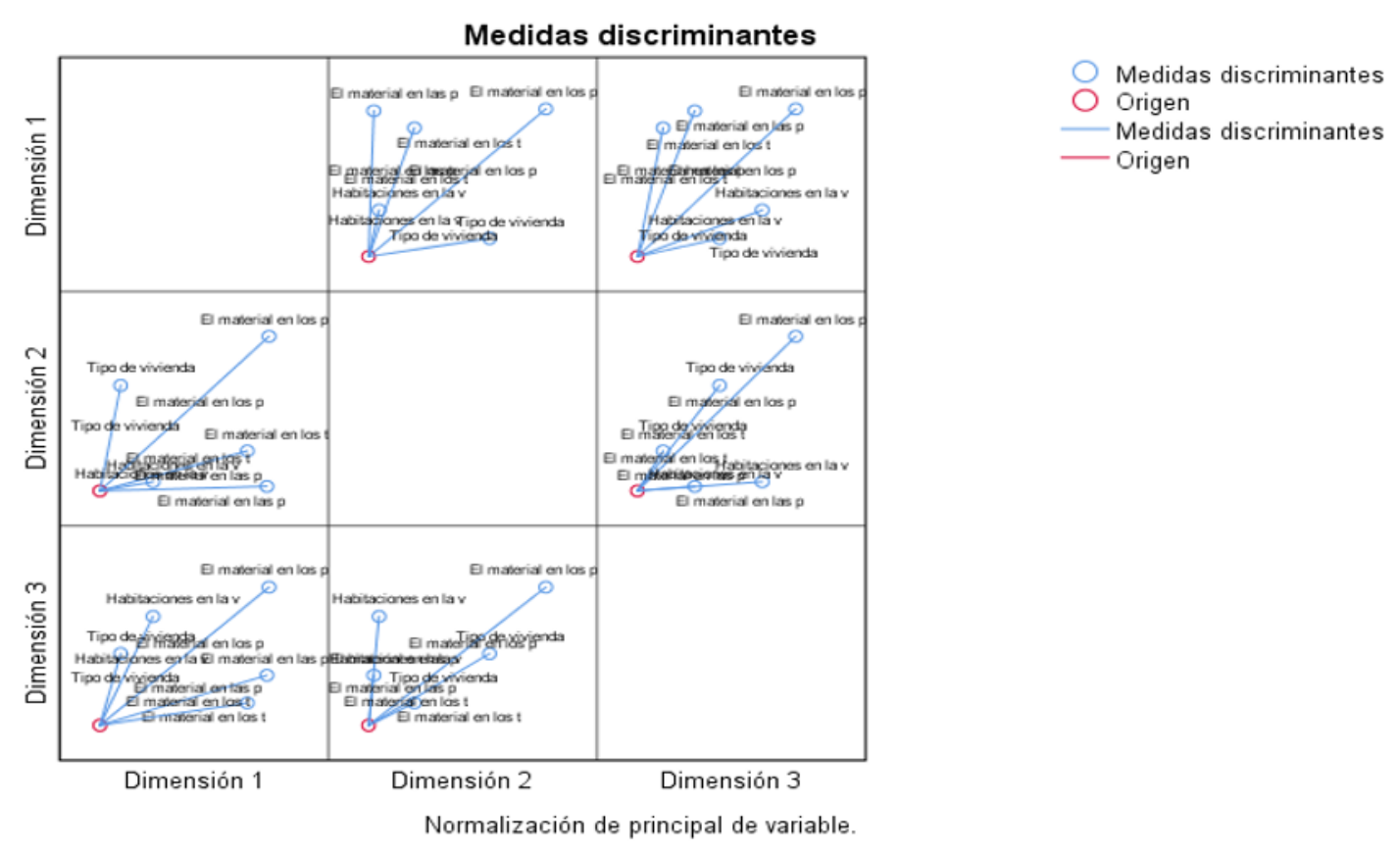

Figura 3. Medidas discriminantes por dimensiones 
Tabla 7

Características de la vivienda y del hogar según servicios, ENAHO 2017

\begin{tabular}{|c|c|c|c|c|}
\hline \multirow[b]{2}{*}{ Dimensión } & \multicolumn{4}{|c|}{ Varianza contabilizada para } \\
\hline & $\begin{array}{c}\text { Alfa de } \\
\text { Cronbach }\end{array}$ & $\begin{array}{c}\text { Total } \\
\text { (autovalor) }\end{array}$ & Inercia & $\%$ de varianza \\
\hline 1 & ,673 & 2,165 & ,433 & 43,295 \\
\hline 2 &, 346 & 1,383 & 277 & 27,660 \\
\hline 3 &, 168 & 1,155 &, 231 & 23,102 \\
\hline Total & & 4,703 & ,941 & \\
\hline Media &, $453^{\mathrm{a}}$ & 1,568 &, 314 & 31,352 \\
\hline
\end{tabular}

a. La media de alfa de Cronbach se basa en la media de autovalor.

En la Tabla 7, del modelo permite observar que se crearon 3 dimensiones. El autovalor cuenta en la proporción de información del modelo que es explicada por cada dimensión; permite analizar la importancia de cada una de ellas. Se puede observar que la primera dimensión es más importante para el modelo que la segunda y la tercera dimensión. Se espera que las dimensiones que se obtienen a raíz de Análisis de correspondencia a mayor dependencia de las variables mayor inercia, esto quiere decir que las categorías presentan mayor dispersión de varianza en la primera dimensión y en las dimensiones 2 y 3 , son parecidas en cuanto a variabilidad. El alfa de Cronbach indica también que tan relacionadas están las variables observables que componen las variables latentes, las dimensiones, por lo que ambas dimensiones tienen una relación directa.

\section{Tabla 8}

\section{Variables transformadas de Correlaciones}

\begin{tabular}{lccccc}
\hline & $\begin{array}{c}\text { Donde } \\
\text { Abastece } \\
\text { de agua }\end{array}$ & $\begin{array}{c}\text { El baño del } \\
\text { hogar está } \\
\text { conectado a }\end{array}$ & $\begin{array}{c}\text { Alumbrado } \\
\text { del hogar }\end{array}$ & $\begin{array}{c}\text { que usan el } \\
\text { hogar }\end{array}$ & $\begin{array}{c}\text { Teléfono } \\
\text { (fijo) }\end{array}$ \\
\hline Donde Abastece de agua & 1,000 &, 755 &, 438 &, 109 &, 189 \\
El baño del hogar está conectado a &, 755 & 1,000 &, 293 &, 166 &, 226 \\
Alumbrado del hogar &, 438 &, 293 & 1,000 &, 078 &, 102 \\
Combustible que usan el hogar &, 109 &, 166 &, 078 & 1,000 &, 231 \\
Su hogar tiene: Teléfono (fijo) &, 189 &, 226 &, 102 &, 231 & 1,000 \\
Dimensión & 1 & 2 & 3 & 4 &, 736 \\
Autovalor & 2,165 & 1,099 &, 775 &, 224 \\
\hline
\end{tabular}

En la Tabla 8, se puede apreciar una alta relación entre el baño o servicio higiénico que tiene su hogar está conectado a y el abastecimiento de agua en su hogar procede de, el más alto y el tipo de alumbrado del hogar: electricidad y el abastecimiento de agua en su hogar procede de, el segundo más alto. 


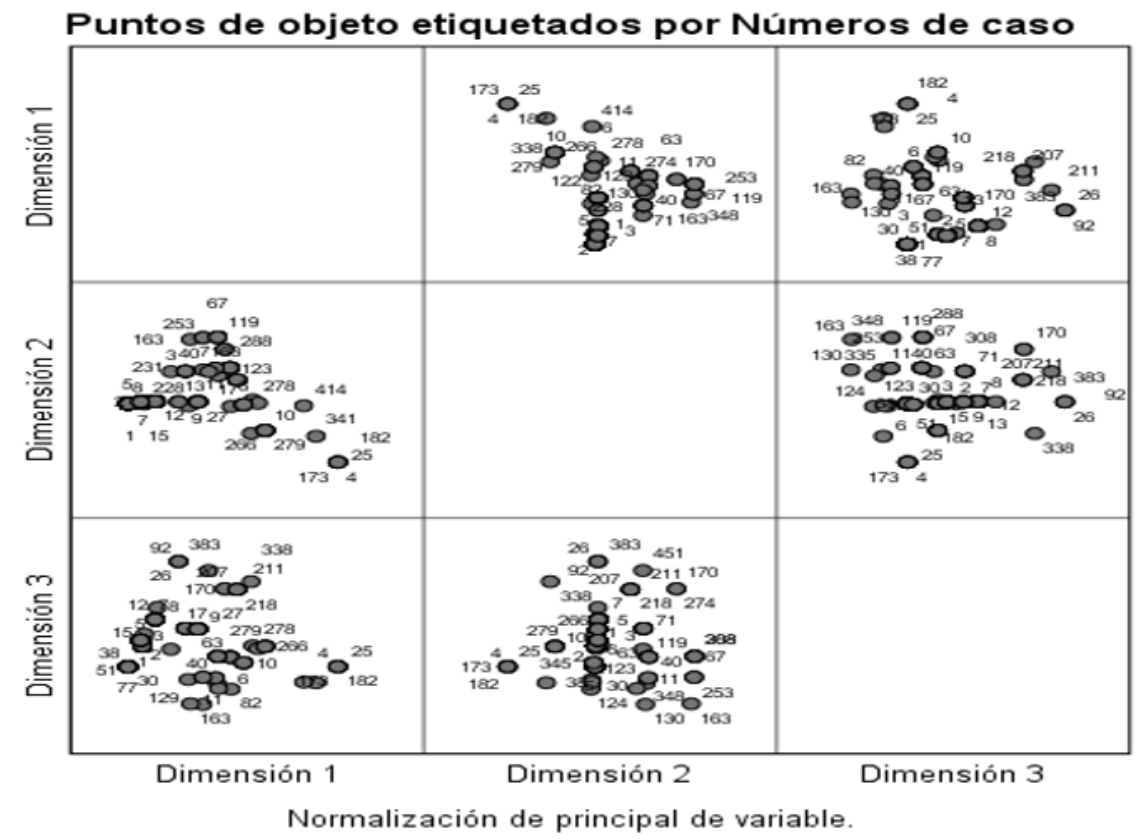

Figura 4. Gráfico de cuantificaciones para la característica de la vivienda y del hogar, según servicios ENAHO 2017.

\section{Tabla 9}

\section{Medidas Discriminantes}

Medidas discriminantes

Variables

\begin{tabular}{lcccc} 
& 1 & 2 & 3 & Media \\
\hline Donde Abastece de agua &, 777 &, 765 &, 379 &, 641 \\
El baño del hogar está conectado a &, 729 &, 423 &, 038 &, 397 \\
Alumbrado del hogar &, 367 &, 188 &, 023 &, 193 \\
Combustible que usan el hogar &, 109 &, 006 &, 405 &, 173 \\
Su hogar tiene: Teléfono (fijo) &, 183 &, 000 &, 310 &, 164 \\
Total, activo & 2,165 & 1,383 & 1,155 & 1,568 \\
\% de varianza & 43,295 & 27,660 & 23,102 & 31,352 \\
\hline
\end{tabular}

En la Tabla 9, se puede analizar en la primera dimensión Donde se abastece de agua que es el más alto, seguido de El baño del hogar está conectado a, luego en la segunda 

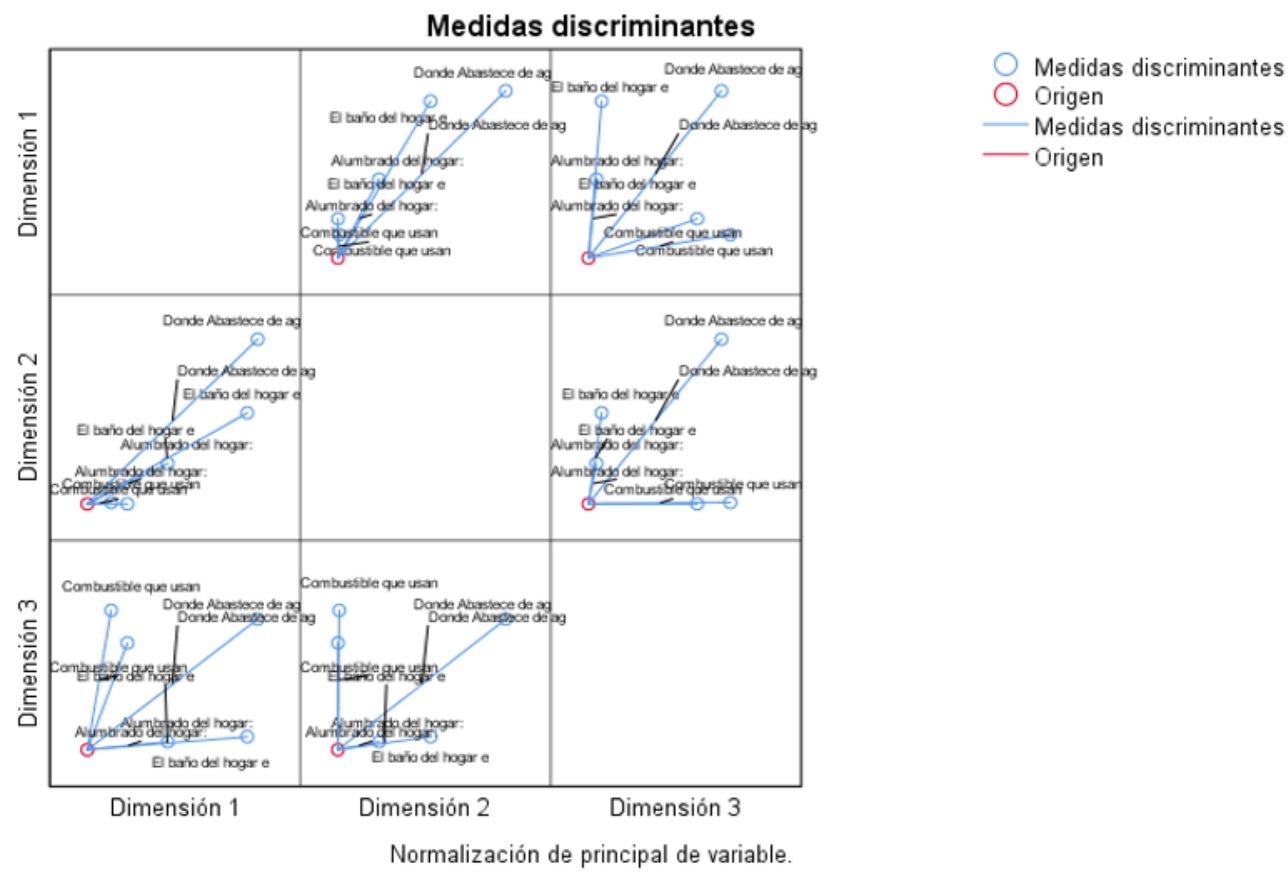

Figura 5. Medidas discriminantes educación, según servicios ENAHO 2017

\section{Tabla 10}

\section{Características de la familia según su educación, ENAHO 2017}

\begin{tabular}{cccc}
\hline \multirow{2}{*}{ Dimensión } & Alfa de Cronbach & \multicolumn{2}{c}{ Varianza contabilizada para } \\
&, 859 & 3,193 & Tnercia \\
\hline 1 &, 757 & 2,534 &, 639 \\
2 &, 651 & 2,086 &, 507 \\
3 & & 7,812 &, 417 \\
Total &, $770^{\text {a }}$ & 2,604 & 1,562 \\
Media & &, 521 \\
\hline
\end{tabular}

a. La media de alfa de Cronbach se basa en la media de autovalor.

En la Tabla 10, podemos observar que el alfa de Cronbach en la dimensión 1 es de 0.859, en la dimensión 2 es 0.757 y en la dimensión 3 es 0.651.
Luego tenemos la inercia para cada dimensión de 0.639, 0.507 y 0.417 , respectivamente. 
Tabla 11

Variables transformadas de Correlaciones

\begin{tabular}{|c|c|c|c|c|c|}
\hline Variables & $\begin{array}{c}\text { Año de } \\
\text { estudios } \\
\text { y nivel } \\
\text { que } \\
\text { aprobó: }\end{array}$ & $\begin{array}{c}\text { ¿Sabe leer y } \\
\text { escribir? }\end{array}$ & $\begin{array}{c}\text { ¿Se } \\
\text { matriculo en } \\
\text { algún centro } \\
\text { de Ed. } \\
\text { básica o } \\
\text { superior? }\end{array}$ & $\begin{array}{l}\text { ¿Cuál es el } \\
\text { grado o año } \\
\text { de estudios }\end{array}$ & $\begin{array}{c}\text { ¿Hizo uso } \\
\text { del servicio } \\
\text { de } \\
\text { Internet? }\end{array}$ \\
\hline Año de estudios y nivel que aprobó: & 1,000 & ,277 & ,347 &,- 015 &, 513 \\
\hline Sabe leer y escribir & ,277 & 1,000 & 033 &,- 013 &, 178 \\
\hline $\begin{array}{l}\text { ¿Se matriculo en algún centro de Ed. } \\
\text { básica o superior? }\end{array}$ & ,347 & ,033 & 1,000 &,- 405 &, 151 \\
\hline ¿Cuál es el grado o año de estudios ${ }^{\mathrm{a}}$ &,- 015 &,- 013 &,- 405 & 1,000 & ,019 \\
\hline ¿Hizo uso del servicio de Internet? &, 513 &, 178 &, 151 & 019 & 1,000 \\
\hline Dimensión & 1 & 2 & 3 & 4 & 5 \\
\hline Autovalor & 1,849 & 1,297 & 872 & ,589 & ,394 \\
\hline
\end{tabular}

a. Los valores perdidos se han imputado con la modalidad de la variable cuantifica

En la Tabla 11, podemos notar que la matriculó en algún centro de educación básica o autocorrelación más alta que corresponde a: superior? y Año de estudios y nivel que aprobó, ¿Hizo uso del servicio de internet? y Año de siendo la primera correlación moderada y la estudios y nivel que aprobó, seguido de ¿Se segunda muy baja, respectivamente.

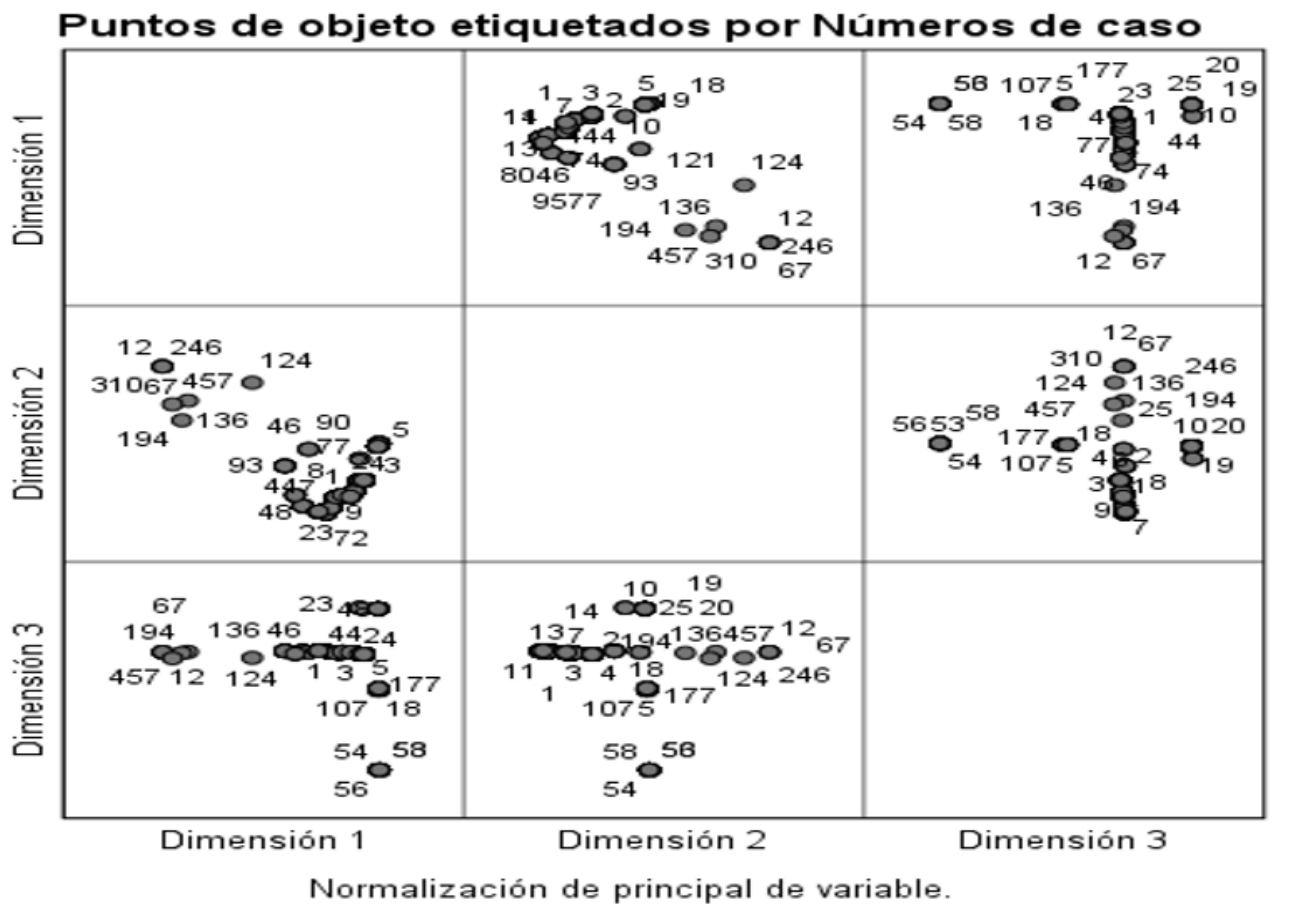

Figura 6. Gráfico de cuantificaciones para educación, según servicios de internet ENAHO 2017. 
Tabla 12

Medidas Discriminantes

\begin{tabular}{lcccc}
\hline & \multicolumn{3}{c}{ Dimensión } \\
& 1 & 2 & 3 & Media \\
\hline Año de estudios y nivel que aprobó: &, 942 & 1,033 & 1,043 & 1,006 \\
¿Sabe leer y escribir? &, 231 &, 436 & 1,041 &, 569 \\
¿Se matriculo en algún centro de Ed &, 694 &, 394 &, 000 &, 363 \\
básica o superior? & & & & \\
¿Cuál es el grado o año de estudios &, 705 &, 361 &, 000 &, 355 \\
¿Hizo uso del servicio de Internet? &, 621 &, 311 &, 001 &, 311 \\
Total, activo & 3,193 & 2,534 & 2,086 & 2,604 \\
\hline
\end{tabular}

En la Tabla 12, se puede distinguir la medida discriminante de la dimensión 1 , años de estudio y nivel que aprobó es el más alto, ¿Cuál es el grado o año de estudios? Es el segundo más alto. Así mismo, en la segunda dimensión se tiene el valor de 1.033 al igual que en la tercera dimensión el valor de 1.043, respectivamente.

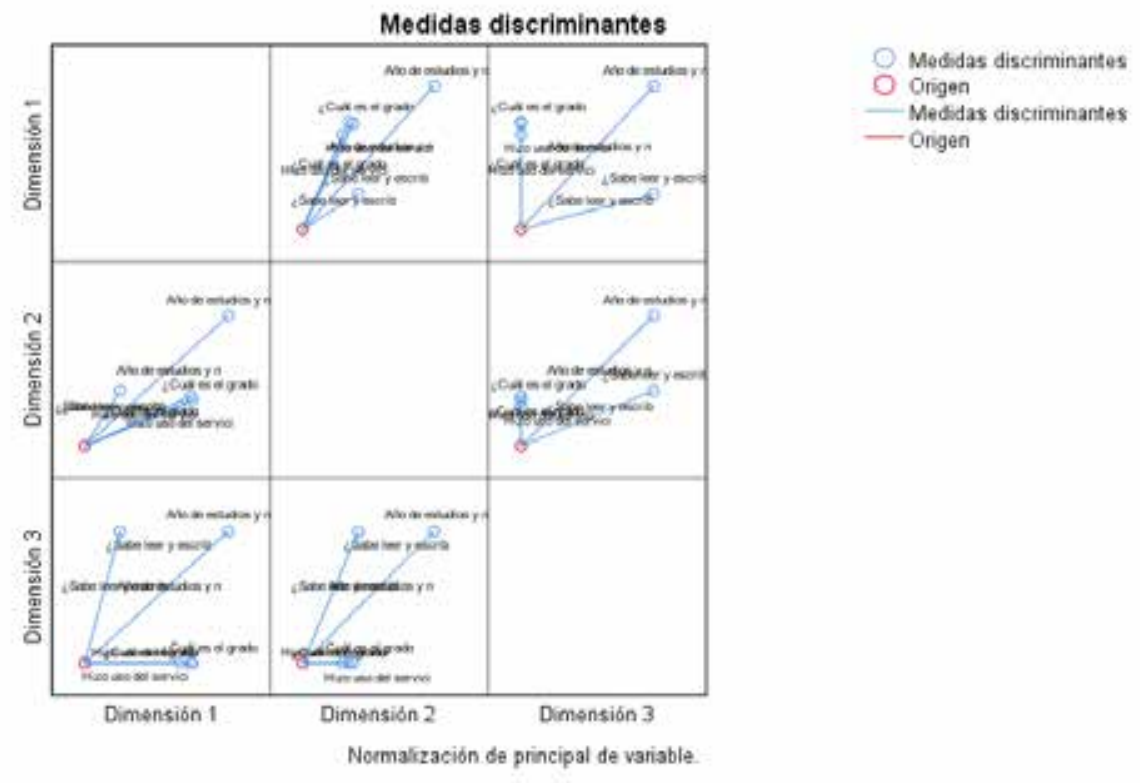

Figura 7. Gráfico de cuantificaciones para educación, según servicios de internet ENAHO 2017.

Tabla 13

Características de la familia según salud, ENAHO 2017

\begin{tabular}{ccccc}
\hline & \multirow{2}{*}{ Alfa de } & \multicolumn{3}{c}{ Varianza contabilizada para } \\
Dimensión & Cronbach & Total (autovalor) & Inercia & \% de varianza \\
\hline 1 &, 169 & 1,092 &, 546 & 54,609 \\
2 &,- 203 &, 908 &, 454 & 45,391 \\
Total & & 2,000 & 1,000 & \\
Media &, $000^{\mathrm{a}}$ & 1,000 &, 500 & 50,000 \\
\hline
\end{tabular}

a. La media de alfa de Cronbach se basa en la media de autovalor. 
La Tabla 13, de resumen del modelo permite observar que se crearon 3 dimensiones. El autovalor de cuenta en la proporción de información del modelo que es explicada por cada dimensión; permite analizar la importancia de cada una de ellas. Se puede observar que la primera dimensión es más importante para el modelo que la segunda dimensión. Así mismo, explica el porcentaje más alto que la segunda, lo cual se espera que las dimensiones que se obtienen a raíz de Análisis de correspondencia a mayor dependencia de las variables mayor inercia, esto quiere decir que las categorías presentan mayor dispersión de varianza en la primera dimensión y en la dimensión 2 , son parecidas en cuanto a variabilidad. El alfa de Cronbach indica también que tan relacionadas están las variables observables que componen las variables latentes, las dimensiones, por lo que ambas dimensiones tienen una relación directa.

\section{Tabla 14}

Relación Que Existe Entre Las Dimensiones Educación Y Las Dimensiones Salud, Bienestar De Servicio Y Bienestar De Infraestructura De Las Familias De La Provincia Del Santa

\begin{tabular}{lccc}
\hline \multicolumn{1}{c}{ Correlación Rho de Spearman } & SALUD & $\begin{array}{c}\text { Bienestar de } \\
\text { Servicio }\end{array}$ & $\begin{array}{c}\text { Bienestar de } \\
\text { Infraestructura }\end{array}$ \\
Educación & 0.005 & $0.576^{* *}$ & $0.567^{* *}$ \\
\hline $\begin{array}{l}\text { Coeficiente de correlación de } \\
\text { Spearman }\end{array}$ & 0.920 & 0.000 & 0.000 \\
Sig. (bilateral) & 477 & 477 & 477 \\
$\mathrm{~N}$ & & & \\
\hline
\end{tabular}

Hipótesis:

Ho: No hay relación entre las dimensiones Educación y Salud, Servicio e Infraestructura de las familias de la provincia del santa

$\mathrm{H1}$ : Si hay relación entre las dimensiones Educación y Salud, Servicio e Infraestructura de las familias de la provincia del santa

En el caso de relación entre las dimensiones Educación y Salud se acepta la hipótesis Nula, el valor $\mathrm{T}=0.108973$ se encuentra en la región de aceptación y el valor $p=0.920$ es mayor a 0.05 , podemos concluir que no existe diferencia significativa y por lo tanto no existe relación entre las dimensiones mencionadas, por otro lado en el caso de las dimensiones Educación y Bienestar de Servicio se acepta la hipótesis nula, el valor $T=15.2378$ se encuentra en la región de aceptación y el valor $\mathrm{p}=0.000$ es menor a 0.05 , podemos concluir que existe diferencia significativa y por lo tanto existe relación entre las dimensiones mencionadas. $Y$, por último, las dimensiones educación y bienestar de infraestructura se acepta la hipótesis nula, el valor $\mathrm{T}=15.0021$ se encuentra en la región de aceptación y el valor $\mathrm{p}=0.000$ es menor a 0.05 , podemos concluir que existe diferencia significativa y por lo tanto existe relación entre las dimensiones de Educación y Bienestar de infraestructura.

Objetivo específico 4: Determinar el Indicador de pobreza multidimensional de la provincia del Santa 2017.

El autor (Peña, 2015) utiliza la siguiente fórmula:

$$
M_{o}=\sum_{i=1}^{n} \sum_{j=1}^{d} g_{i j}^{0} / n d
$$


Donde:

$\mathrm{H}$ : Tasa de pobreza multidimensional.

$g_{i j}^{0}=$ elementos de la matriz de privaciones.

A: La privación promedio compartida entre los pobres $q=$ número de hogares pobres

$\mathrm{n}=$ número de hogares analizados

Mo o IPM: Índice de pobreza multidimensional $\mathrm{d}=$ número de dimensiones consideradas.

Tabla 15

Índice de pobreza multidimensional (IPMACM)

\begin{tabular}{lcccc}
\hline & & Dimensión & & \\
& 1 & 2 & 3 & Media \\
\hline Tipo de Vivienda &, 239 &, 772 &, 032 &, 347 \\
Material predominante paredes &, 599 &, 120 &, 548 &, 422 \\
Material predominante pisos &, 507 &, 075 &, 157 &, 246 \\
Material predominante techos &, 409 &, 058 &, 185 &, 217 \\
Número de habitaciones &, 250 &, 045 &, 150 &, 148 \\
El abastecimiento de agua &, 565 &, 891 &, 494 &, 650 \\
Baño en su hogar conectado &, 640 &, 906 &, 227 &, 591 \\
Tipo de Alumbrado electricidad &, 135 &, 054 &, 163 &, 117 \\
Combustible Usan Hogar Cocinar &, 100 &, 056 &, 016 &, 057 \\
Alimentos & & & & \\
Teléfono &, 222 &, 034 &, 018 &, 091 \\
Total activo & 3,665 & 3,010 & 1,990 & 2,889 \\
\hline
\end{tabular}

A continuación, se narra el procedimiento asociado a la construcción del IPM con ayuda de la técnica multivariante de Análisis de correspondencia múltiple, IPMACM.

Se está considerando las variables antes presentadas en la tabla: tipo de vivienda, material predominante en las paredes, material predominante en pisos, techos, número de habitaciones, el abastecimiento del agua, baño en su hogar conectado a, tipo de alumbrado eléctrico, combustible que usan en el hogar para cocinar alimentos y si tiene teléfono.

En base a estas variables se realiza un nuevo análisis sin las variables cuyos valores son muy pequeños como tipo de vivienda, número de habitaciones, tipo de alumbrado electricidad y si tiene teléfono 
Tabla 16

Medidas Discriminantes

\begin{tabular}{lcccc}
\hline & \multicolumn{3}{c}{ Dimensión } & \multirow{2}{*}{ Media } \\
\hline Material predominante paredes & 1 & 2 & 3 & \\
Material predominante pisos &, 724 &, 151 &, 600 &, 491 \\
Material predominante techos &, 573 &, 006 &, 073 &, 217 \\
El abastecimiento de agua &, 598 &, 031 &, 227 &, 252 \\
Baño en su hogar conectado a &, 664 &, 940 &, 577 &, 682 \\
Total activo & 2,987 & 2,073 & 1,838 &, 361 \\
\hline
\end{tabular}

Con el propósito de establecer la fórmula para IPMACM se utilizarán las medidas discriminantes de la primera dimensión:

IPMACM $=0.724$ * Mat_Paredes +0.573 * Mat_Pisos +0.498 * Mat_techos +0.528 * Abas_agua +0.664 * Baño_conec $=5.446$

\section{CONCLUSIONES}

Del análisis correspondiente, se evaluó la pobreza multidimensional en cuatro niveles de pobreza aplicando el método de las necesidades básicas insatisfechas, cuando el nivel No pobres quiere decir que el $1.7 \%$ cuenta con todas las necesidades básicas, cuando es regularmente pobre que es el $85.5 \%$ si al menos no cuenta con una necesidad básica, para el nivel Pobre que es el $12.4 \%$ quiere decir que al menos no cuenta con 2 o 3 necesidades básicas y cuando se dice Pobre extremo que representa $0.4 \%$ quiere decir que viven en escenarios difíciles en la Provincia del Santa.

Al analizar la relación que existe entre las dimensiones Educación y Salud de las familias de la provincia del Santa, tenemos como indicador al coeficiente Rho de Spearman con un valor de 0.092, el cual nos indica que no existe relación entre las dos dimensiones antes mencionadas. Al analizar la relación que existe entre las dimensiones Educación y Bienestar de Infraestructura de las familias de la provincia del Santa, tenemos como indicador al coeficiente Rho de Spearman con un valor de 0.005, el cual nos indica que no existe relación entre las dos dimensiones antes indicadas.
Al analizar la relación que existe entre las dimensiones Educación y Bienestar de Servicios de las familias de la provincia del Santa, tenemos como indicador al coeficiente Rho de Spearman con un valor de 0.567, el cual nos muestra que existe una relación moderada entre las dos dimensiones antes mostradas.

El índice de pobreza multidimensional es de 5.446 que viene a ser el valor de corte, si el PCMACM $>5.446$, el hogar clasifica como pobre, en caso contrario es no pobre.

\section{REFERENCIAS BIBLIOGRÁFICAS}

Atkinson, A. B. (1987). On the measurement of poverty. Econometría, 749-764.

Barr, N. (2005). Economics of the Walfare State. Oxford: 2012.

Boltvinik, J. (2013). Medición multidimensional de pobreza. América Latina de precursora a rezagada. Sociedad y Equidad, 4-29.

Castañeda, C. P. (2018). Perú es sexto en la Región en la Pobreza Multidimensional. La Cámara, 6-8. 
Urbina, M. R. (2017). La pobreza monetaria desde la perspectiva de la pobreza multidimensional: el caso peruano. Enfoque n.0s, 77 - 98.

Peña, L. A. (2015). Un indicador de pobreza multidimensional alternativo para venezuela. Innovaciencia, 29 - 40.

Benvin, E. R. (2016). Propuesta de un indicador de bienestar multidimensional de uso del tiempo y condiciones de vida aplicado a Colombia, el Ecuador, México y el Uruguay . Revista CEPAL 118, 122 - 145.

Foster, S. A. (2008). Recuento y medición multidimensional de la pobreza. OPHI WORKING PAPER SERIES.

Kaztman, R. (1989). La heterogeneidad de la pobreza. El caso de Montevideo . Revista de la Cepal, 142-152.

López, E. (2005). Problemas sociales desigualdad pobreza y exclusion social. Madrid: Biblioteca Nueva.

Díaz, M. A. (2012). Análisis Estadístico de Datos Multivariados. Bogotá D. C., Colombia: Universidad Nacional de Colombia - Sede Bogotá.

ONU. (1995). Cumbre Mundial, sobre desarrollo social. Implementación y seguimiento de los acuerdos de la Cumbre Social Post Ginebra, 1-18.

Peña, E. A. (2015). Un indicador de Pobreza multidimensional alternativo para Venezuela. Innovaciencia, 52 - 74.

Pinzón, R. C. (2011). Ïndice de pobreza Multidimensional para Colombia. ARCHIVOS DE ECONOMÍA.

Pinzón, R. C. (2011). Índice de Pobreza Multidimensional para Colombia (IPMColombia) 1997- 2010. Archivos de Economía.
Sen, A. (1979). Issues in the Measurement of Poverty. Journal of Economics, 286 - 307. 